\title{
Design, Integration and Testing of Compliant Gripper for the Installation of Helical Bird Diverters on Power Lines
}

\author{
Inmaculada Armengol*, Alejandro Suarez*, Guillermo Heredia*, and Anibal Ollero*
}

\begin{abstract}
The installation and removal of bird diverters from power lines is conducted nowadays by human operators working from manned helicopters or from the power line itself, which entails a certain risk and cost that can be reduced if an aerial manipulator performs these tasks. This paper presents the design of a lightweight gripper (70 $\mathrm{g})$ which is specific for the installation of helical bird diverters. It consists of a claw-type compliant mechanism that is integrated in an anthropomorphic dual arm system, which is intended to perform the operation, and is attached to a multirotor through a long-reach pendulum configuration. The paper also covers the mechanical integration as well as the utilization of a teleoperation system to test the gripper for the installation at a test bench.
\end{abstract}

Index Terms - Aerial Manipulator, Gripper, Helical Bird Flight Diverter.

\section{INTRODUCTION}

The development of aerial robots with manipulation capabilities, also known as aerial manipulators [1][2], enables the possibility to perform inspection and maintenance tasks in difficult access areas such as power lines, pipe structures or solar plants. The expected reduction in time and cost, along with the increase in safety, has motivated the research carried out in this field over the last decade [3][4][5].

The inspection and maintenance of the power grid is a particularly suitable application example in which aerial manipulation robots are useful due to the vast extension and high altitude and risk of such workspace. One of these tasks is the installation of bird flight diverters, which are devices that are used to avoid the collision and electrocution of birds with the power lines. There are two main type of devices, the helical and the clip diverters, as shown in Figure 1a. Currently, their installation and removal are performed by human operators, which involves certain risks for the workers' safety. In the case of the installation of helical bird diverters, the operator has to work from a helicopter or from the line itself. Safety in the execution of maintenance operations can be increased by means of employing a teleoperated or autonomous aerial manipulator (Figure 1.b). This way the person in charge of the procedure can operate safely from a ground control station (GCS), eliminating the risk of human harm from happening because of accidents.

Once the risk for humans is removed, safety and reliability requirements must also be considered in the development of an aerial robotic manipulator. The interactions between the robot and the environment during the installation could entail hazardous situations for the infrastructures or the own platform and manipulator. One way to diminish the danger of impact of the multirotor with the power line is the use of a long reach

*Inmaculada Armengol (inmarmmor@gmail.com), Alejandro Suarez (asuarezfm@us.es), Guillermo Heredia (guiller@us.es) and Anibal Ollero configuration [6][7], where the distance between the aerial platform and the manipulator, and therefore the obstacles, is increased. This setup also leads to a reduction of the incident downwash from the multirotor on the arms and the device to install. Another improvement consists of including mechanical compliance in both the arms [8][9] and the grippers [10][11] [12] to be able to withstand impacts and overloads as well as accommodating to position deviations due to control errors or wind disturbances.

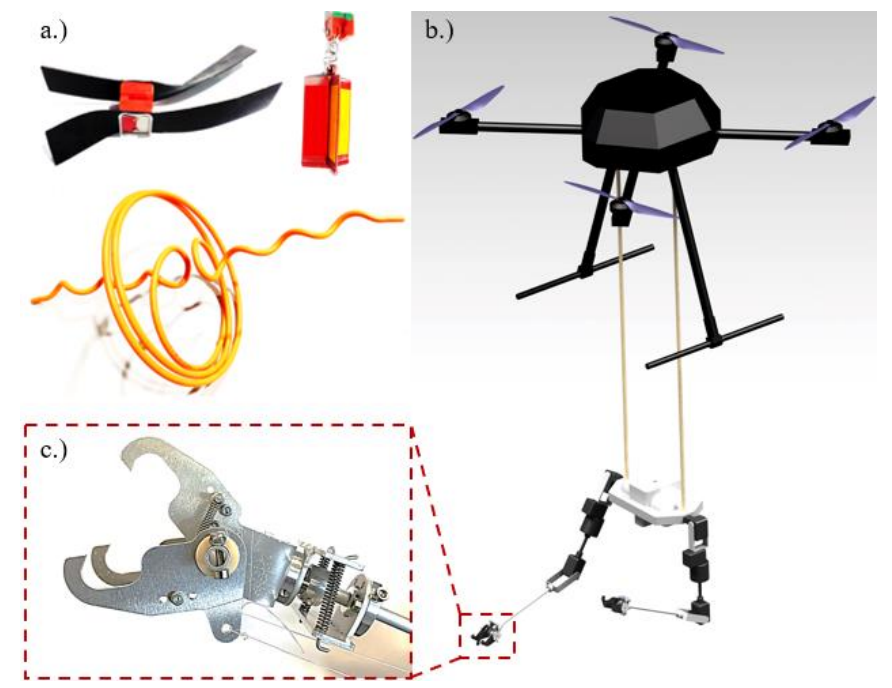

Figure 1. Overview of the bird diverters (a), the installation configuration (b) and the final design of the gripper (c).

Several prototypes of manipulators [1][2][6][8] and grippers [13][14] for aerial manipulation have been developed in previous works. The configuration of the aerial manipulator used for the installation of the helical device is similar to the one appearing in [6], where an arm is attached to a hexarotor through a one-meter-length pendulum. However, in this paper a two-arm system like the one developed in [8] is employed. Other possible manipulation schemes are described in [1] and [2]. In the first one, Suseong K. et al. develop a manipulator integrating a quadrotor with a 2-DOF (Degree of Freedom) robotic arm, and in the second one the system consists of an unmanned helicopter with a 7-DOF industrial robotic arm.

Literature on gripper design and grasping mechanisms includes from simple claw configurations [15] to more dexterous prototypes with a higher number of DOFs [16][17], with mechanisms varying from tendon-driven actuation [10] to grabbing by suction [18] or magnetic devices [18][19], among others. An overview of these technologies is presented in [13] and [14], with the former reference focusing on soft

(aollero@us.es) are with the GRVC Robotics Labs at the University of Seville. Camino de los descubrimientos, s/n. E-41092 Seville. 
grippers. Due to payload limitations in the aerial platform and the robotic manipulator, a simple but effective and reliable solution is desirable to allow the grasping and installation of the helical bird diverter, with few DOFs and a compact and lightweight construction.

The main contribution of this paper is the development and experimental validation of a gripper mechanism that can be used to perform the installation of helical bird diverters on power lines. The gripper consists of three aluminum frames and support shafts that implement a claw-type compliant mechanism that adapts its shape to the device to ensure a robust grasping, as shown in Figure 1.c. This adaptability is possible due to a pair of independent extension springs that are employed to maintain a closed-by-default configuration. The gripper is attached to the forearm of the anthropomorphic dual arm through a passive and compliant wrist joint that allows the rotation in the forearm link axis (pronation/supination) and it is tendon-driven by a smart servo actuator. The gripper is validated with a teleoperation task in a fixed-base test bench before the performance of flight test.

The rest of the paper is organized as follows. Section II presents the design requirements in terms of the device's geometry, the installation procedure and the integration in the manipulator. Section III describes the design of the gripper, including the procedure and the frame design along with the union to the forearm. The application for the installation of the device is described in depth in Section IV, whereas Sections V and VI cover the experimental results and the conclusion and future work, respectively.

\section{DESIGN REQUIREMENTS}

\section{A. Diverter Geometry}

The installation of the helical bird diverter will be carried out with the LiCAS A1 [20], an anthropomorphic, lightweight and compliant dual arm system developed by LiCAS Robotic Arms. The arms will be equipped with a grasping mechanism that must enable a reliable and robust grip of the device, whose geometry and shape will be studied in the first place.

The device to manipulate is a $0.6 \mathrm{~kg}$ PVC (Polyvinyl Chloride) helix which is partly rigid and partly flexible. Figure 2 shows an image of the diverter with its overall dimensions. The extreme branches of the diverter can be treated as a rigid solid, but the central and wider part can change its size in such a way that the total length of the object ranges from 0.86 to $1.10 \mathrm{~m}$. The complex geometry of the device makes necessary for the gripper to be able to accommodate to its shape when

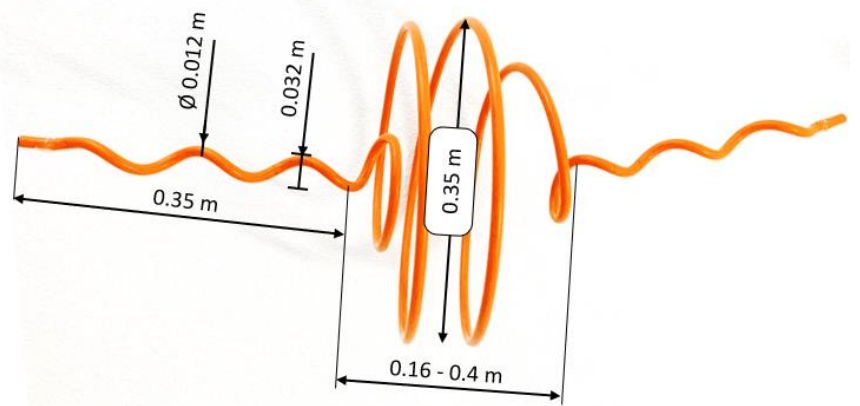

Figure 2. Helical device dimensions. grabbed and allow the arms to grasp the object at the different points according to the installation procedure, described in next subsection.

\section{B. Installation Steps}

After analyzing from video sequences [21] and laboratory tests (Figure 3) how human workers perform the installation of the diverters on the power lines, it has been established that the installation procedure can be divided into five steps:

Step 1. The arms grab the diverter and perform a clockwise rotation to set the object into a vertical position.

Step 2. To pass the helix over the power line, the vertical separation between the wrists is increased by elevating the left arm and moving it forwards to introduce the first coil.

Step 3. Once the object is over the line, this step consists in wrapping the central branch. While the right arm remains holding the right branch, the left arm lets go of the diverter and then grabs it again under the line to bring it around the wire. This is repeated twice.

Step 4. With the central branch settled, the arms focus on the installation of the left branch. The procedure is similar to the one in the previous step but changing the grasping points and relying on wrist/fingers dexterity. The left side must be grabbed closer to its edge to increase the leverage effect.

Step 5. The last task is settling the right branch, just as it is done in Step 4 for the opposite side, but symmetrically.

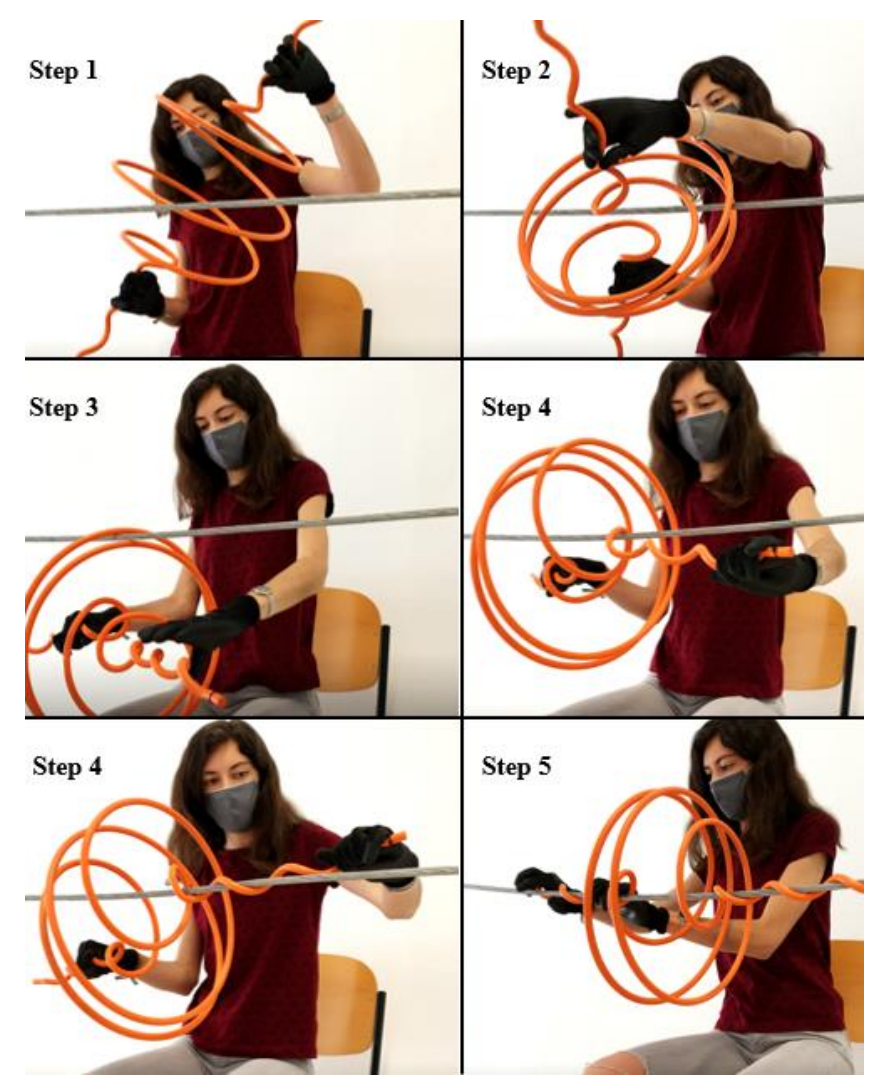

Figure 3. Installation steps (sequence attached). 


\begin{tabular}{|l|c|}
\hline Wheelbase [mm] & 610 \\
\hline Propellers [inch] & $21 \times 7$ \\
\hline Weight (with batteries) [kg] & 6 \\
\hline MTOW [kg] & 9.5 \\
\hline LiPo Battery [mAh] & $12 \mathrm{~S}, 7000$ \\
\hline Max. flight time [min] & 45 \\
\hline Motors & DJI E2000 \\
\hline
\end{tabular}

Table 1. Proskytec LRM specifications.

This analysis allowed the identification of certain design specifications for the gripper. As most of the time the arms are holding the diverter, a closed-by-default mechanism can be beneficial to reduce the energy consumption of the driving servo. This way, the gripper servos are actuated only in the few moments when the arms have to release the device. The installation experience shows that the difficulty increases in terms of dexterity and force as the branches are rolled around the cable (Steps 4-5), particularly when the branch has to be grasped behind the cable and pulled backwards. This requires a certain degree of rotation in the wrist or fingers to catch the device, which should be grasped as close as possible to its tip to reduce the effort required in its rotation. Additionally, the holding arm (right, Step 4) must exert a certain moment on the branch while the operation arm (left, Step 4) is performing the installation to avoid that the whole device rolls around the power line. Finally, in terms of time performance, although time is not prioritized in this initial study, and just to give an idea of the human performance, it takes about $15 \mathrm{~s}$ for a trained human operator to install the device [21].

\section{Integration with the Aerial Platform}

As stated in the introduction, the aerial manipulation robot intended to conduct the installation task is composed of an anthropomorphic dual arm robotic system integrated into a multirotor in long reach configuration. Unlike our previous work [6][7], where the long reach link consisted of two flexible aluminum profiles, here a novelty is introduced, and two strings are used to bind the arms with the platform to facilitate the landing and reduce the lateral torques induced over the multirotor base. The aerial platform is a Proskytec LRM quadrotor [22], whose main features are listed in Table 1. The robotic arms [20] are an evolution of previous prototype described in [8], implementing the same kinematic configuration with 4-DOF for end effector positioning.

The main requirements derived from this subsection are the weight and flight time. The whole configuration (pendulum - arms - grippers - diverter) cannot exceed the maximum payload of the quadrotor, and the installation must be carried out within the available time associated to the battery capacity. Therefore, the grippers must be as light and compact as possible. In addition, the integration of the grippers has to be done according to the preexisting frame structure of the arms, which is described in Subsection III-C.

\section{GRIPPER DESIGN}

\section{A. Design Procedure}

The design of the gripper mechanism is an iterative and empirical process driven by the experimental evaluation of the effectiveness of the developed solutions.

The evolution of the models designed for grasping the helical bird diverter is shown in Figure 4. The first prototype was a tendon driven actuator that consisted of three humanlike fingers attached to a base frame, all parts 3D-printed in PLA (Polyactic Acid). Nylon filaments were tied to the tip of the fingers and driven to a pulley screwed to a servo. This design was discarded due to two main reasons. On the one hand, the use of thin adhesive tape with plastic layers to bind the fingers' phalanges with each other resulted in weak links unable to withstand the loads reached during the installation. On the other hand, the fingers are opened by default, and as indicated in Section II, during the installation procedure the grippers remain closed most of the time, so this configuration was inefficient in that regard.

The conclusions drawn from the first prototype led to a complete change in the design concept, introducing a claw configuration with a rigid frame structure. This second gripper is made of three carbon fiber frames, each of them attached to

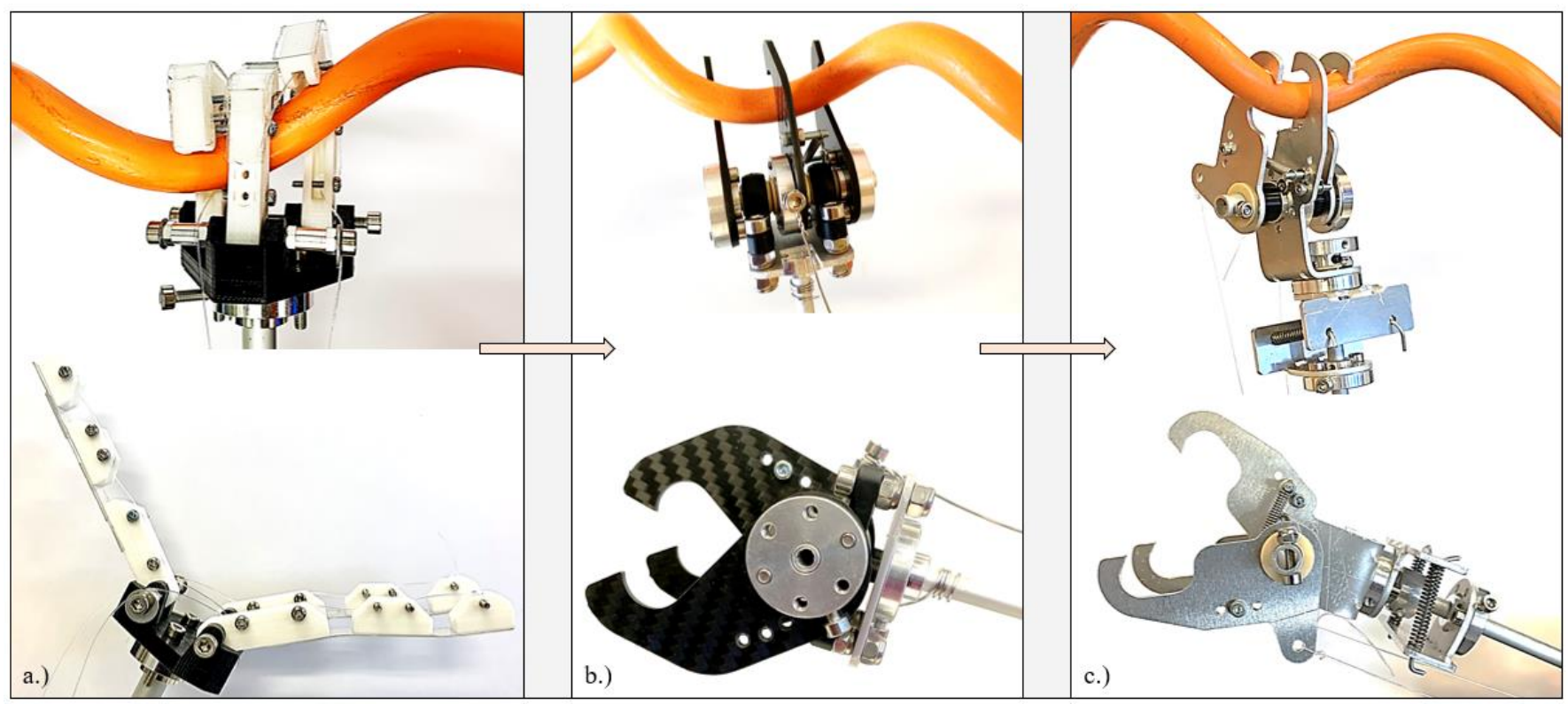

Figure 4. Evolution of prototypes. 
coaxial hubs with an aluminum shaft that goes through them, as shown in Figure 4.b. The shaft allows the rotation of the upper frame to open the gripper while the other two remain at a fixed position attached to their respective hubs. A closed by default configuration is achieved with an extension spring that is connected to both the middle and one of the side frames. It is important to highlight the fact that in this case both the left and right frames are connected and rotate simultaneously, as only one spring is used. Although this approach improved the reliability of the grasping, there were still two unresolved issues. First, in those steps of the installation procedure in which the helical diverter has to be stretched or set in a vertical position, there was slippage. Second, for certain grasping poses, only one of the lateral frames of the gripper contacted the device because of the curved shape of the helix and the lack of accommodation of the two frames.

In order to overcome such difficulties and improve the design in terms of weight, an additional design (see Figure 4.c) was developed using the previous prototype as a basis. In this implementation, the aluminum hubs attached to the lateral frames are removed, allowing for a reduction in frame weight, and removing the pillow bearings of the base, which are no longer needed because the middle frame is directly attached to the forearm through a passive and compliant wrist joint that allows the pronation and supination rotation. Another improvement is the independent actuation of each lateral frame with two extension springs, ensuring that the contact happens in both sides.

\section{B. Frame Design}

The frame design accounts for the requirements imposed by the geometry of the problem and the installation procedure. While for the second prototype only one frame was designed, for the final configuration the shape was parametrized as a function of the grasping distance $d$, that can take the values $[20,30,40] \mathrm{mm}$, as it can be seen in Figure 5. The final design has two types of frames: the side frame (SF) and the middle frame (MF), both made of $2 \mathrm{~mm}$ laser-cut aluminum sheets, where each gripper has two SFs and one MF.

Each SF is driven by the gripper servo, so an anchor point is needed to join the two frames with the servo through nylon filaments, as described in the next subsection. This point is a 3 $\mathrm{mm}$ drill separated $25 \mathrm{~mm}$ from the SF's central axis. The closing drills have a $2.5 \mathrm{~mm}$ diameter and the bolts that go through them hold the extension springs that are responsible a.)

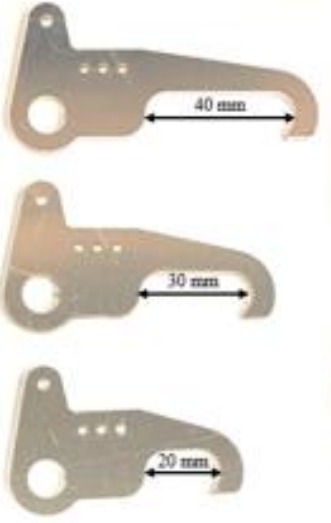

b.)

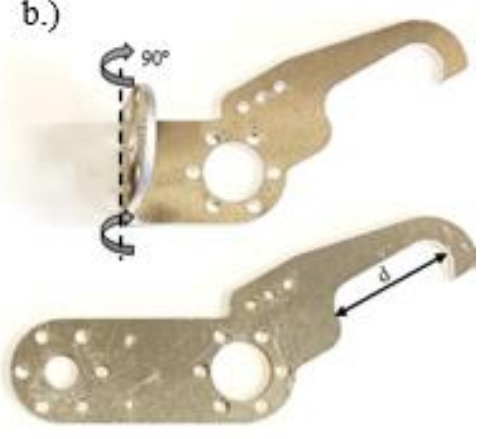

Figure 5. a.) Side frames. b.) Middle frame. for the close-by-default arrangement. As for the MF, its fixed orientation led to a 30-degree phase gap with respect to the horizontal plane, so that when the gripper opens the device is grabbed without the wrists having to elevate its position. In addition, it should be noted that the MF is designed in such a way that it can be directly attached to the wrist with a 90degree fold of the piece, as portrayed in Figure 5.b.

\section{Integration in the Forearm}

The two most important aspects of the integration (see Figure 6) of the gripper with the rest of the arm are the nylon SF-servo connections and the passive and compliant wrist joint. Compared to steel wires, the nylon string reduces the stress felt by the servo exclusively to those time intervals in

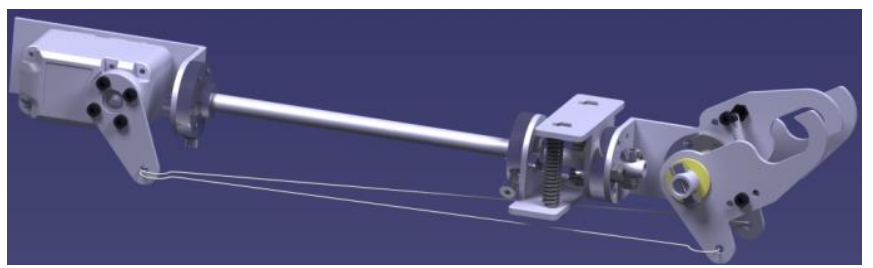

Figure 6. CAD representation of the gripper integrated in the forearm.

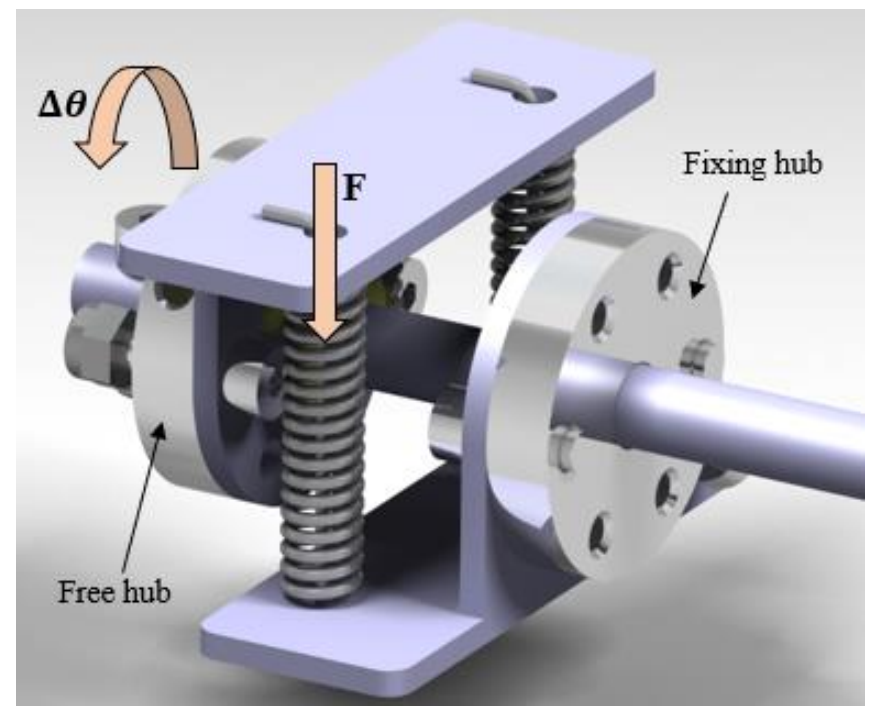

Figure 7. Detailed view of the compliant wrist joint mechanism.

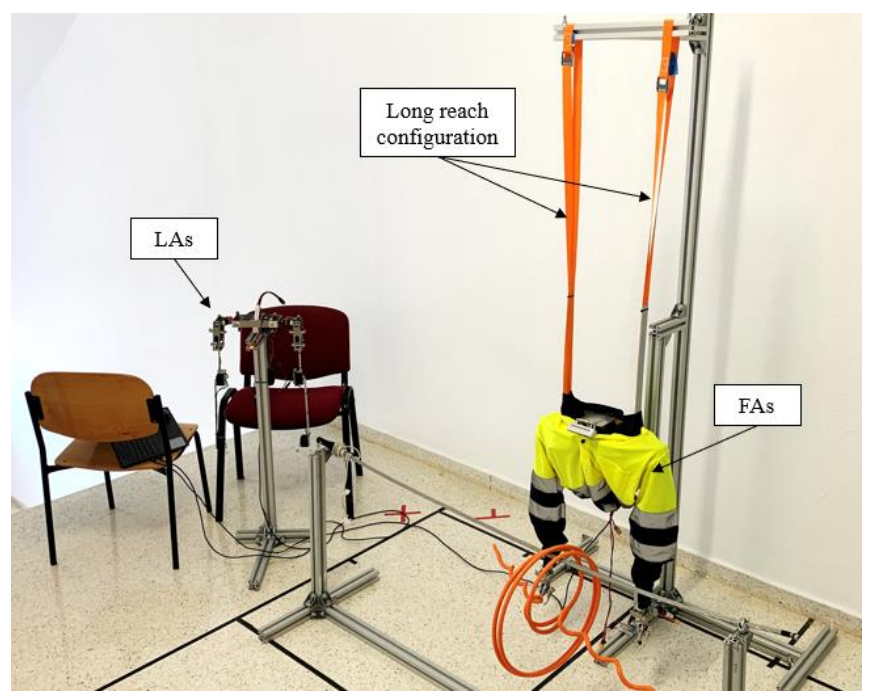

Figure 8. Experimental setup with leader arms (LAs) and follower arms (FAs) in long reach configuration. 
which the gripper is opened, as this material does not possess compressive strength. As for the wrist joint, it is a spring-lever compliant mechanism that allows the accommodation of the gripper around the forearm axis, with two springs in charge of supporting the torques exerted during the installation of the helical bird diverter (see Figure 7). The joint is built with two compression springs, two hubs, two aluminum wires and two L-shaped frames manufactured by folding 90 degrees a lasercut aluminum flat frame, similarly to the MF described in the previous subsection. One of the hubs is fixed to the forearm link, whereas the other allows the rotation of the gripper.

\section{APPLICATION FOR THE INSTALLATION OF THE HELICAL BIRD DIVERTER}

As the final goal of the developed gripper is to allow the installation of the helical bird flight diverter, the validation experiment consists of installing the device from Steps 1 to 3 (see Section II-B). Because of the reasons detailed at the end of Section II-B, steps 4 and 5 are left for future research. This section describes how the installation is carried out along with the experimental setup.

\section{A. Teleoperation Scheme}

The method proposed in this paper to test the reliability of the designed gripper and at the same time take the first steps to install the helical diverter is a preliminary teleoperated system where two anthropomorphic dual arm systems are employed. One of them is referred to as Leader Arms (LAs) and the other as Follower Arms (FAs). The FAs manipulate the helical device and are commanded by the LAs, replicating the joint motions of the LAs, moved by a human operator. This corresponds to the external joint control scheme described in [7], using an UDP socket to send the joints' references from the LAs to the FAs. Such reference values are obtained directly from the servos' feedback, avoiding the need for kinematic solutions. The teleoperation scheme enables a remote installation where the operator can be safely placed in a ground control station. Since this work is focused on identifying and reproducing the installation procedure, we simplify the experimental setup by placing the operator next to the FAs to avoid the need of additional interfaces. The communication between the LAs and the FAs are handled in localhost, using a GCS laptop for both manipulators.

\section{B. Experimental Setup}

The experimental setup is depicted in Figure 8. On the left side, the human operator seats on the red chair to control the GCS and manipulate the LAs. On the right, the FAs are suspended from a two-meter height structure in long reach configuration through a pair of safety harnesses, so that the installation experience is similar to the one considered for the

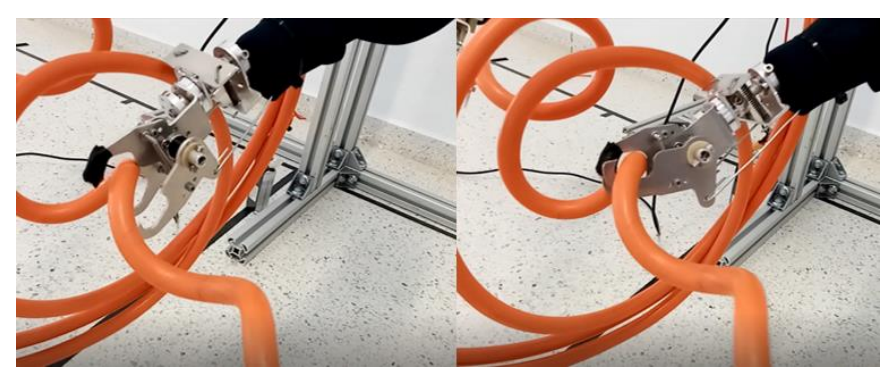

Figure 9. Left arm grasping the device. aerial manipulator (Figure 1.b). The long reach link consists of two $30 \mathrm{~cm}$ length aluminum profiles and two adjustable strings that are attached to the arms' shoulder frame and the top of the structure. This combination provides the advantages of both materials: the aluminum profiles act as lever, reducing the reaction pitch rotation experienced by the arms when they lift the helical diverter, and the strings increase the flexibility and entail the advantages described in Section II-C.

The LAs are mounted on a $90 \mathrm{~cm}$ height structure to offer a comfortable experience for the human operator, who remains seated during the realization of the task. The position of the FAs relative to the power line is determined empirically taking into account the reach and workspace of the arms [20], as well as the installation procedure described in Section II-B. It was determined that the power line should be at $32 \mathrm{~cm}$ from the base of the arms (X-axis), and $25 \mathrm{~cm}$ under the shoulder (Zaxis).

\section{EXPERIMENTAL RESULTS}

This section presents the results obtained in the execution of the experiment described in Section IV.

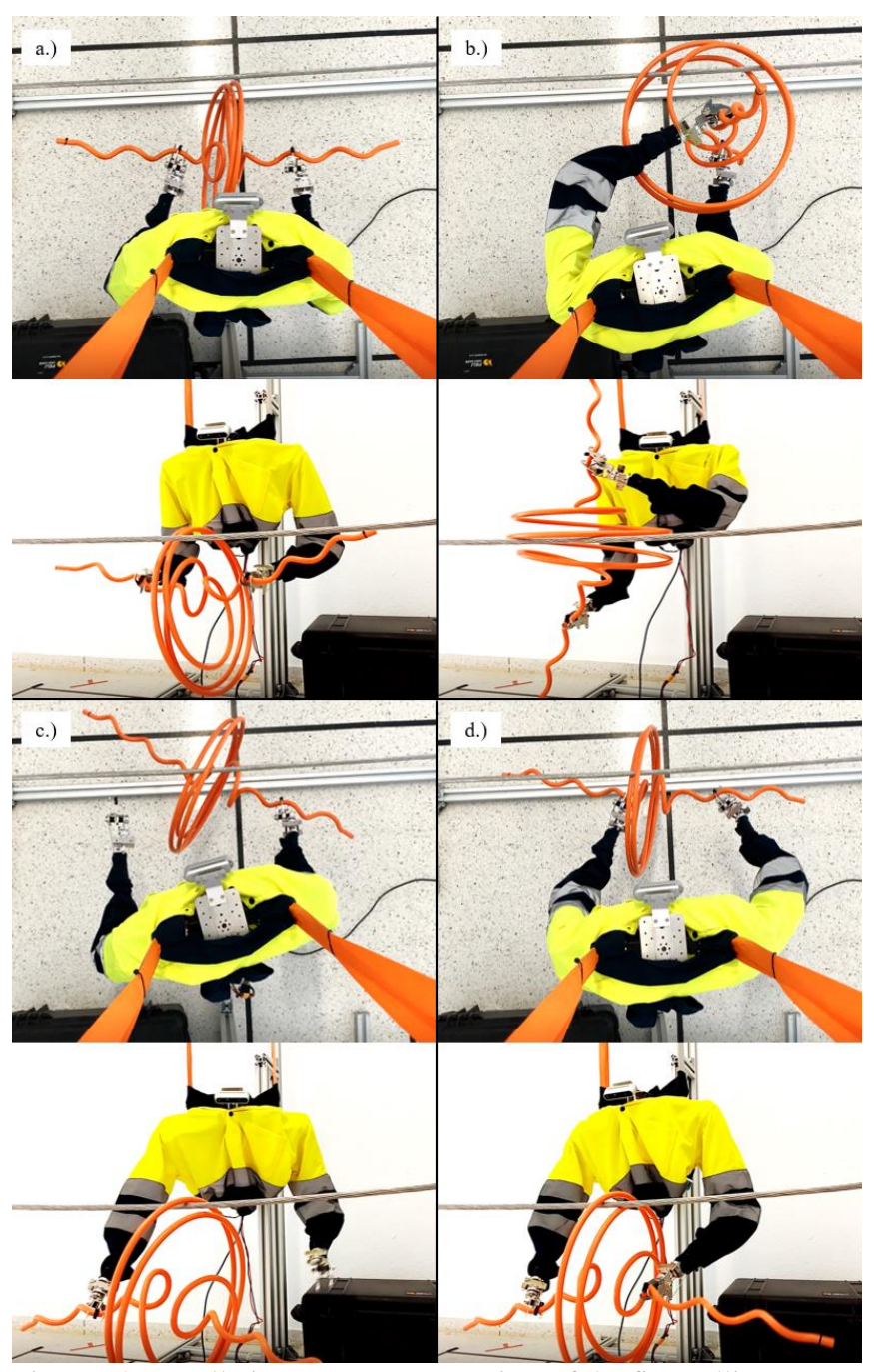

Figure 10. Installation sequence, overview of the first rolling around the power line, including the top and frontal view. 


\section{A. Gripper Validation}

The goal of this experiment is to validate the effectiveness of the developed gripper through the installation of the helical bird diverter in testbench, proving that the gripper can not only hold the device (see Figure 9) but also maintain the grip during the manipulation. The installation video, which includes a close sequence of the grasping, can be found in [23]. Figure 10 shows a sequence from both the top and frontal view. These images depict how the arms pass the helical diverter over the line and perform the first roll around it. Once the grippers hold the device in an initial resting position (Figure 10.a), the arms are moved to turn the helical diverter into a vertical position and pass the object over the line (Figure 10.b). After that, the left arm releases the device and comes back to grab it from under the power line and execute another rolling (Figures 10.c and 10.d). Even in the most challenging situation for the gripper, which is the instant when the left arm rotates the diverter to achieve a vertical position, the design is effective, as the helical device does not fall and the installation can be performed. As a result of this experiment, it is also observed that the left gripper is the one to "do the hard work", while the right gripper is relegated to holding still and orienting the right branch.

The analysis of the video evidences the eventual contact between the arms and the power line during the realization of the installation operation. It is necessary to remark that the experimental results presented in [24] show that the electrical interaction between a high voltage $(15 \mathrm{kV})$ power line and an aerial manipulator may affect the aerial platform due to the electrostatic discharge, whereas the robotic manipulator is not.

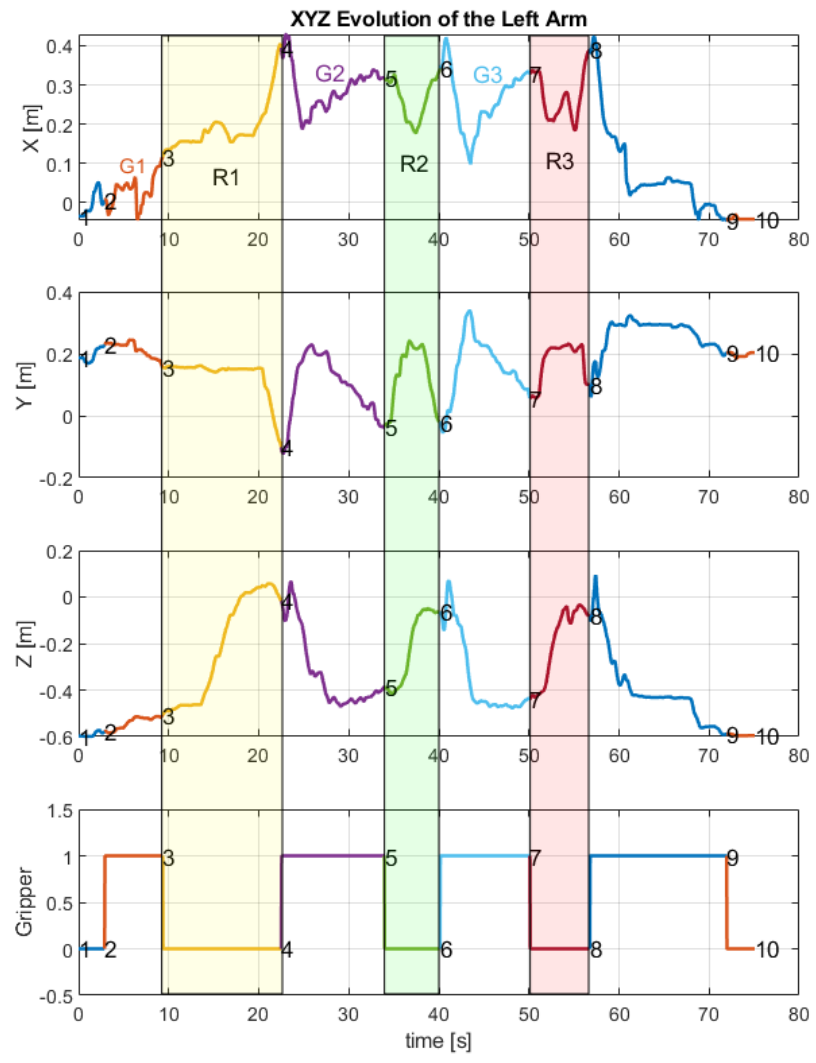

Figure 11. X, Y, Z and Gripper values of the left arm.

\section{B. Installation Procedure Highlights}

Although the main goal of the experiment was to validate the grippers, it is also interesting to analyze the results derived from the installation procedure. To do this, the joint variables

3D Installation Trajectory

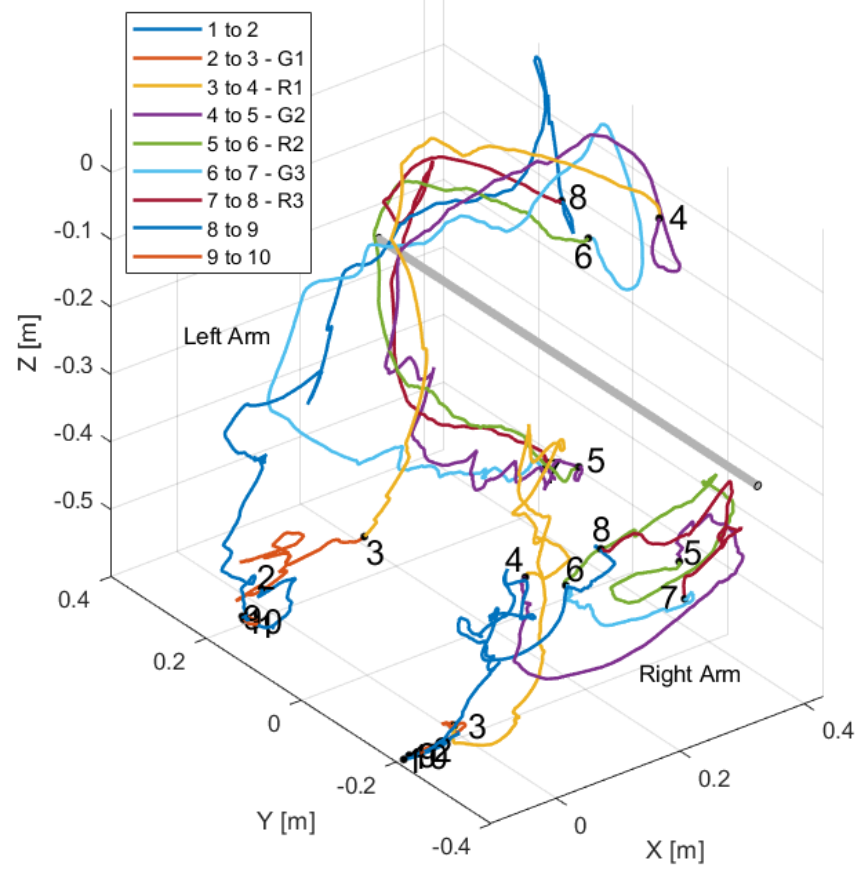

Figure 12. Segmented 3D representation of the arms' trajectory during the experiment.

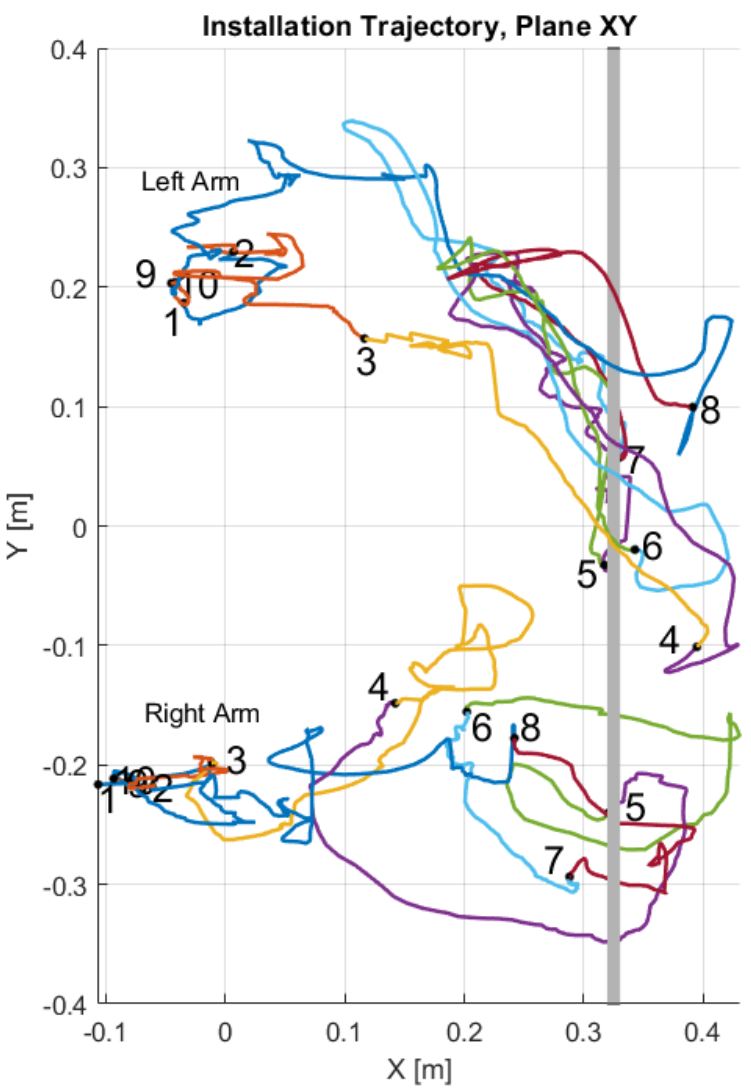

Figure 13. Segmented representation of the arms' trajectory projected on the XY plane. 


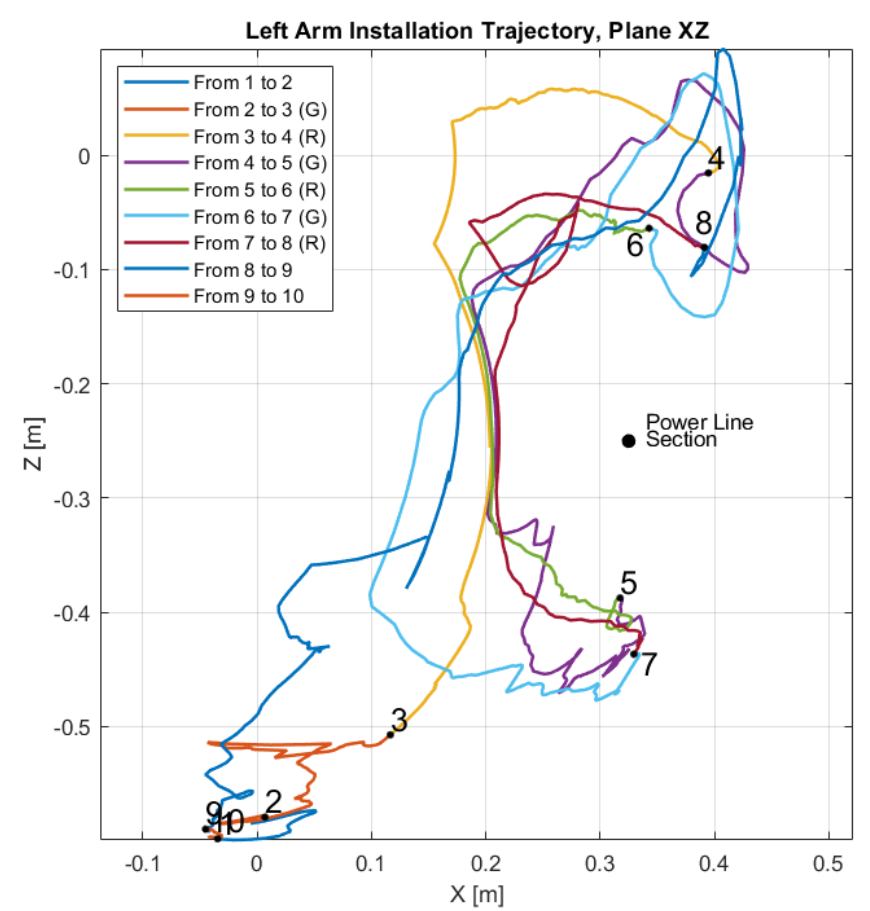

Figure 14. Segmented representation of the left arm trajectory projected on the $\mathrm{XZ}$ plane.
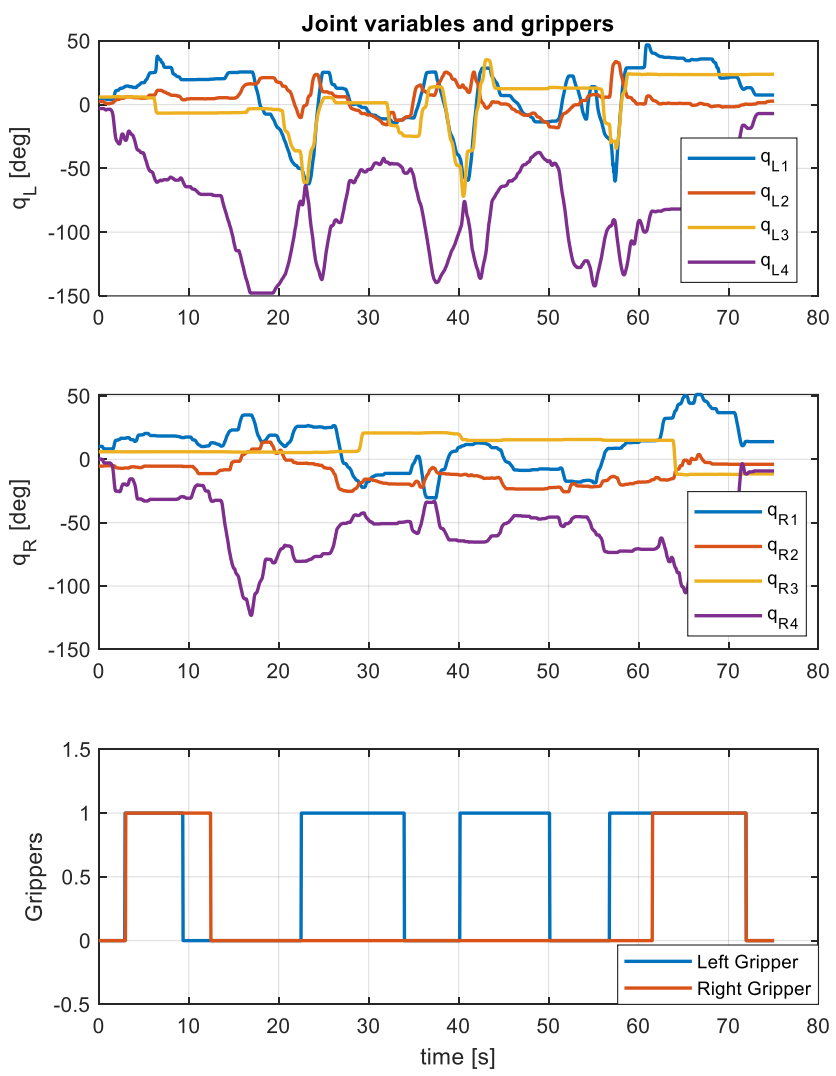

Figure 15. Joint variables and gripper states of both the left and right arm.

of the FAs were logged during the teleoperation experiment and processed to better understand the patterns and phases of the installation steps performed in Steps 1-3.

As stated in the previous subsection, the left arm is the key in this first part of the procedure, so the trajectory of the arms' end effectors is divided into a set of phases that depend on the state of the gripper: every time the gripper is actuated, a segment ends and a new one begins. This way, besides the initial and final segments of approach and withdrawal, there are two types of movements, the grasping $(\mathrm{G})$ and the rolling (R) segments. This division is made to favor a recognition of patterns in the installation and an easier transition into an autonomous installation, which is the next step in this research. Figure 11 shows the cartesian positions of the left arm as a function of time, as well as the state of its gripper, which is open for 1 and closed for 0 . The described segments are depicted in this image, and the trajectories are shown in Figures 12, 13 and 14, with a 3D view and projections on the $\mathrm{XY}$ and $\mathrm{XZ}$ plane, respectively. Some patterns are observed in these images, the evolution of $\mathrm{Z}$ in G2 and G3 is quite similar, and the same applies to X. In Figure 14, it can be appreciated how the yellow, green and red segments, which correspond to the rolling procedures, have the same evolution when $\mathrm{Z} \in[-$ $0.3,-0.2] \mathrm{m}$.

The cartesian variables, represented first for a better understanding of the paths followed by the arms, are calculated from the joint variables (see Figure 15). These variables are, from $q_{1}$ to $q_{4}$, the shoulder pitch, shoulder roll, shoulder yaw and elbow joints [8].

\section{CONCLUSION AND FUTURE WORK}

This paper presented the design and validation of a lightweight gripper mechanism that is in charge of grabbing and holding the helical bird diverter during its installation on power lines. After three design iterations, the effectiveness of the final configuration has been proven by an experiment that consisted in performing the first steps of the installation of the device in long reach configuration in a teleoperated way.

As future work, it is considered the autonomous installation employing a vision system. Nevertheless, firstly the research will be focused on the study of the whole installation procedure, not only steps 1 to 3 , and it will be determined whether an improvement of the current gripper features in terms of slippage, reach and/or wrist dexterity are needed. In addition, a more thorough teleoperation system will be developed, considering the delays associated to the wireless communications between the ground control station and the operation arms.

\section{ACKNOWLEDGMENT}

This work is supported by the AERIAL-CORE project (H2020-2019-871479) funded by the European Commission.

\section{REFERENCES}

[1] S. Kim, S. Choi and H. J. Kim, "Aerial manipulation using a quadrotor with a two DOF robotic arm," in IEEE/RSJ International Conference on Intelligent Robots and Systems, Tokyo, Japan, 2013, pp. 4990-4995.

[2] K. Kondak et al., "Aerial manipulation robot composed of an autonomous helicopter and a 7 degrees of freedom industrial manipulator," in IEEE International Conference on Robotics and Automation (ICRA), Hong Kong, China, 2014, pp. 2107-2112.

[3] F. Ruggiero, V. Lippiello and A. Ollero, "Aerial Manipulation: A Literature Review," in IEEE Robotics and Automation Letters, vol. 3, no. 3, July 2018, pp. 1957-1964. 
[4] X. Meng, Y. He, and J. Han, "Survey on Aerial Manipulator: System, Modeling, and Control," in Robotica, vol. 38, no. 7, 2020, pp. 12881317.

[5] A. Ollero et al., "The AEROARMS project: aerial robots with advanced manipulation capabilities for inspection and maintenance," in IEEE Robotics \& Automation Magazine, vol. 25, no. 4, December 2018, pp. 12-23.

[6] A. Suarez, P. Sanchez-Cuevas, M. Fernandez, M. Perez, G. Heredia and A. Ollero, "Lightweight and Compliant Long Reach Aerial Manipulator for Inspection Operations," in IEEE/RSJ International Conference on Intelligent Robots and Systems (IROS), Madrid, Spain, 2018, pp. 67466752.

[7] A. Suarez, F. Real, V. M. Vega, G. Heredia, A. Rodriguez-Castaño and A. Ollero, "Compliant Bimanual Aerial Manipulation: Standard and Long Reach Configurations," in IEEE Access, vol. 8, 2020, pp. 8884488865 .

[8] A. Suarez, P. R. Soria, G. Heredia, B. C. Arrue and A. Ollero, "Anthropomorphic, compliant and lightweight dual arm system for aerial manipulation," in IEEE/RSJ International Conference on Intelligent Robots and Systems (IROS), Vancouver, BC, Canada, 2017, pp. 992-997.

[9] Yu She, Deshan Meng, Hongliang Shi and Hai-Jun Su, "Dynamic modeling of a 2D compliant link for safety evaluation in human-robot interactions," in IEEE/RSJ International Conference on Intelligent Robots and Systems (IROS), Hamburg, Germany, 2015, pp. 3759-3764.

[10] A. Suarez, G. Heredia and A. Ollero, "Compliant and Lightweight Anthropomorphic Finger Module for Aerial Manipulation and Grasping," in Reis L., Moreira A., Lima P., Montano L., MuñozMartinez V. (eds) (Robot 2015: Second Iberian Robotics Conference. Advances in Intelligent Systems and Computing), Springer, vol 417, 2015.

[11] Wang, J. and Lan, C., "A Constant-Force Compliant Gripper for Handling Objects of Various Sizes," in Journal of Mechanical Design, July 2014, pp. 071008:1-10.

[12] A. Suarez, G. Heredia and A. Ollero, "Lightweight compliant arm with compliant finger for aerial manipulation and inspection," in IEEE/RSJ International Conference on Intelligent Robots and Systems (IROS), Daejeon, Korea (South), 2016, pp. 4449-4454.

[13] Sabnis, Nilesh. , "A Review: State of The Art of Robotic Grippers," in International Journal of Engineering and Technology, May 2018.

[14] Shintake, J., Cacucciolo, V., Floreano, D. and Shea, H, "Soft Robotic Grippers," in Advanced Materials, vol 30, issue 29, May 2018.

[15] Freyr Erlingsson, B. et. Al, "Axiomatic Design of a Linear Motion Robotic Claw with Interchangeable Grippers", in Procedia CIRP, vol 53, 2016, pp. 213-218.

[16] Z. Xu, V. Kumar and E. Todorov, "A low-cost and modular, 20-DOF anthropomorphic robotic hand: Design, actuation and modeling," in IEEE-RAS International Conference on Humanoid Robots, 2015, pp. 368-375.

[17] Zhe Xu and E. Todorov, "Design of a highly biomimetic anthropomorphic robotic hand towards artificial limb regeneration," in IEEE International Conference on Robotics and Automation (ICRA), Stockholm, Sweden, 2016, pp. 3485-3492.

[18] A. Koivikko et al., "Magnetically switchable soft suction grippers," in Extreme Mechanics Letters, vol. 44, April 2021.

[19] R. Debanik, "Development of novel magnetic grippers for use in unstructured robotic workspace," in Robotics and Computer-Integrated Manufacturing, vol. 35, October 2015, pp. 16-41.

[20] LiCAS A1 - Human-like movements, last accessed May 2021: https://www.youtube.com/watch? $\mathrm{v}=\mathrm{jKQB} 7 \mathrm{NI} 9 \mathrm{M} 7 \mathrm{~g} \& \mathrm{t}=8 \mathrm{~s}$

[21] Bird Diverter Installation, last accessed May 2021: https://www.youtube.com/watch?v=0Iu20ZsuEI4

[22] Proskytec Home Page: https://proskytec.com/

[23] Helical Diverter Installation Experiment: https://youtu.be/KpcuylGl5xE

[24] A. Suarez, R. Salmoral, P. J. Zarco-Periñan, A. Ollero, "Experimental evaluation of aerial manipulation robot in contact with $15 \mathrm{kV}$ power line: shielded and long reach configurations," in IEEE Access, vol. 9, pp. 94573-94585, 2021. 